\title{
Ability of The Cardiorespiratory System to Adapt to Exercise is Impaired in Individuals With Severe Post-Stroke Fatigue
}

\section{Kazuaki Oyake}

Shinshu University

\section{Yasuto Baba}

Tokyo Bay Rehabilitation Hospital

\section{Yuki Suda}

Tokyo Bay Rehabilitation Hospital

Jun Murayama

Tokyo Bay Rehabilitation Hospital

\section{Ayumi Mochida}

Tokyo Bay Rehabilitation Hospital

Yuki Ito

Tokyo Bay Rehabilitation Hospital

Honoka Abe

Tokyo Bay Rehabilitation Hospital

Kunitsugu Kondo

Tokyo Bay Rehabilitation Hospital

\section{Yohei Otaka}

Fujita Health University

Kimito Momose ( $\sim$ kmomose@shinshu-u.ac.jp )

Shinshu University

\section{Research Article}

Keywords: oxygen, FSS, fatigue

Posted Date: December 1st, 2020

DOl: https://doi.org/10.21203/rs.3.rs-112240/v1

License: (a) (i) This work is licensed under a Creative Commons Attribution 4.0 International License.

Read Full License 
1 Ability of the cardiorespiratory system to adapt to exercise is impaired in individuals

2 with severe post-stroke fatigue

3

4 Kazuaki Oyake, PT, Ph.D., ${ }^{1,2}$ Yasuto Baba, PT, B.Sc., ${ }^{2}$ Yuki Suda, PT, B.Sc., ${ }^{2}$ Jun Murayama,

5 PT, B.Sc., ${ }^{2}$ Ayumi Mochida, PT, B.Sc., ${ }^{2}$ Yuki Ito, PT, B.Sc., ${ }^{2}$ Honoka Abe, PT, B.Sc., ${ }^{2}$

6 Kunitsugu Kondo, MD, Ph.D., ${ }^{2}$ Yohei Otaka, MD, Ph.D., ${ }^{2,3}$ Kimito Momose, PT, Ph.D. ${ }^{1 *}$

7

$8{ }^{1}$ Department of Physical Therapy, School of Health Sciences, Shinshu University, Nagano,

9 Japan

$10{ }^{2}$ Department of Rehabilitation Medicine, Tokyo Bay Rehabilitation Hospital, Chiba, Japan

$11{ }^{3}$ Department of Rehabilitation Medicine I, School of Medicine, Fujita Health University,

12 Aichi, Japan

13

14 *Corresponding author: Kimito Momose, PT, Ph.D.

15 Department of Physical Therapy, School of Health Sciences, Shinshu University

16 3-1-1 Asahi, Matsumoto, Nagano 390-8621, Japan

17 Tel: +81-263-37-3453; Fax: +81-263-37-3453

E-mail: kmomose@shinshu-u.ac.jp 


\section{Abstract}

Physical deconditioning after stroke may induce post-stroke fatigue. However, research on this association is limited. Our primary objective was to investigate the associations of poststroke fatigue with oxygen uptake $\left(\dot{\mathrm{VO}}_{2}\right)$ at peak exercise and the time constant of $\dot{\mathrm{V}} \mathrm{O}_{2}$

24 kinetics $\left(\tau \dot{\mathrm{V}} \mathrm{O}_{2}\right)$ at the onset of exercise. The secondary objective was to examine the associations between fatigue and cardiorespiratory variables potentially affecting $\dot{\mathrm{V}} \mathrm{O}_{2}$ during exercise. Twenty-three inpatients from a subacute rehabilitation ward were enrolled in this study. The median (interquartile range) Fatigue Severity Scale (FSS) score as a measure of fatigue was $32(27,42)$ points. The FSS score was not associated with $\dot{\mathrm{V}}_{2}$ at peak exercise during a symptom-limited graded exercise test $(\mathrm{rho}=-0.264 ; \mathrm{p}=0.224)$, while it was significantly associated with $\tau \dot{\mathrm{V}} \mathrm{O}_{2}$ during a submaximal constant-load exercise test (rho $=$ 0.530; $\mathrm{p}=0.009)$. A higher FSS score was also significantly correlated with a longer time constant of cardiac output kinetics ( $\mathrm{rho}=0.476, \mathrm{p}=0.022$ ). These results suggest that the ability of the cardiorespiratory system to adapt to exercise is impaired in individuals with severe post-stroke fatigue. Our findings can contribute to the development of an appropriate rehabilitation program for such individuals. 

energy that is perceived by the individual to interfere with usual or desired activities' [1,2]. In addition, a case definition for post-stroke fatigue included a criterion for self-reported significant fatigue that interfered with daily activities [3,4]. A systematic review reported that the prevalence of post-stroke fatigue could range between $25 \%$ and $85 \%$ [2]. Post-stroke fatigue is associated with various factors, such as depressive symptoms and functional disability [1]. Furthermore, individuals with post-stroke fatigue are reported to have poor recovery of activities of daily living [5,6], a lower rate of returning to work [7], reduced health-related quality of life [8], and increased mortality [6,9]. The underlying pathophysiology of post-stroke fatigue is not completely understood, although probable factors include low excitability of the motor cortex and inflammatory responses [1]. In addition, there is insufficient evidence regarding the effectiveness of rehabilitative exercise programmes for improving post-stroke fatigue [1,10], although there is sufficient evidence that exercise training can improve fitness, balance, mobility, and activities of daily living in individuals with stroke [11]. Post-stroke fatigue has been suggested to be triggered by physical deconditioning,

54 which may lead to the avoidance of physical activities and further deconditioning [12]. However, there is limited evidence on the association between post-stroke fatigue and 
cardiorespiratory fitness [12]. Oxygen uptake $\left(\mathrm{V}_{2}\right)$ at peak exercise measured during a symptom-limited graded exercise test is widely accepted as an indicator of cardiorespiratory capacity in individuals with stroke $[11,13,14] . \dot{\mathrm{V}} \mathrm{O}_{2}$ at peak exercise in individuals with stroke is $26 \%-87 \%$ of that in healthy age- and sex-matched individuals [13]. However, a cross-sectional study in individuals with chronic stroke $(4.1 \pm 3.5$ years post-stroke) reported that post-stroke fatigue was associated with depressive symptoms but not with $\dot{\mathrm{V}}_{2}$ at peak exercise [15]. The assessment of $\dot{\mathrm{V}} \mathrm{O}_{2}$ kinetics at the onset of submaximal exercise has also been shown to provide objective information on cardiorespiratory fitness in individuals with stroke $[16,17]$. Transient measurements of $\dot{\mathrm{VO}}_{2}$ to a constant-load exercise at an intensity below the ventilatory threshold are classified into three phases. The time constant of $\dot{\mathrm{VO}}_{2}$ in phase II $\left(\tau \dot{\mathrm{V}} \mathrm{O}_{2}\right)$ has often been used to assess $\dot{\mathrm{V}} \mathrm{O}_{2}$ kinetics at the onset of exercise, which reflects the ability of the cardiorespiratory system to adapt from rest to a new steady-state during submaximal exercise [18]. A longer $\tau \dot{\mathrm{V}} \mathrm{O}_{2}$ is associated with poorer health status, ageing, and a sedentary lifestyle $[18,19]$. Tomczak et al. [16] reported that $\tau \dot{\mathrm{V}} \mathrm{O}_{2}$ was greater in individuals with stroke than in age-, sex-, and activity-matched healthy adults. $\tau \dot{\mathrm{V}} \mathrm{O}_{2}$ has been reported to be more sensitive than $\dot{\mathrm{VO}}_{2}$ at peak exercise in assessing cardiorespiratory functional changes associated with training and inactive lifestyle [19-22]. Therefore, we hypothesised that the severity of post-stroke fatigue could be associated more strongly with $\tau \dot{\mathrm{V}} \mathrm{O}_{2}$ than $\dot{\mathrm{VO}}_{2}$ 
at peak exercise.

Wu et al. [4] proposed a conceptual model of post-stroke fatigue that biological

factors may trigger fatigue present at an early stage after stroke (usually within the first 3

months after stroke), whereas fatigue present at a later stage after stroke (usually over 1 year

after stroke) may be more attribute to psychological and behavioural factors. However, the

association between post-stroke fatigue at the early stage after stroke and cardiorespiratory

fitness has not been reported. The primary objective of this study was to examine whether

more severe fatigue was associated with a longer $\tau \dot{\mathrm{V}} \mathrm{O}_{2}$ at the onset of exercise measured

during a submaximal constant-load exercise test rather than a lower $\dot{\mathrm{V}}_{2}$ at peak exercise

obtained during a symptom-limited graded exercise test in inpatients at a subacute

rehabilitation ward. In addition, impairments of respiratory and cardiac function to supply

oxygen and the inability of skeletal muscles to extract oxygen may limit the increase in $\dot{\mathrm{V}} \mathrm{O}_{2}$

87 during exercise in individuals with stroke $[16,23,24]$. Thus, our secondary objective was to

identify associations between post-stroke fatigue and cardiorespiratory variables potentially

affecting $\dot{\mathrm{V}} \mathrm{O}_{2}$ during exercise such as the oxygen uptake kinetics efficiency slope (OUES), cardiac output $(\mathrm{CO})$, and arterial-venous oxygen difference ( $\mathrm{AVO}_{2}$ diff). Although there have been no published studies regarding the cardiorespiratory mechanisms underlying the impairments of $\tau \dot{\mathrm{V}} \mathrm{O}_{2}$ in individuals with stroke, ventilatory efficiency and muscle oxygen extraction, measured by the OUES and $\mathrm{AVO}_{2}$ diff, respectively, are lower in individuals with 
94 stroke than in healthy adults $[25,26]$. Therefore, we hypothesised that the impairment of these

95 variables would also be associated with post-stroke fatigue. Elucidating cardiorespiratory

96 factors associated with post-stroke fatigue could contribute to the development of an

97 appropriate rehabilitation program for individuals with post-stroke fatigue.

98

$99 \quad$ Results

100 Participants

101

A flow chart of participants enrolled in the study is shown in Fig. 1. Thirty

individuals with stroke provided informed consent. However, two participants refused to

perform exercise tests. In addition, in five of 28 participants who performed the submaximal

constant-load exercise test, cardiorespiratory data during the test could not be measured

because of technical difficulties. Consequently, 23 participants were included in the analysis.

Although all participants were recruited from a subacute rehabilitation ward, five participants

were in the chronic phase of stroke recovery ( $\geq 3$ months after stroke) [27]. Table 1 shows the characteristics of the participants.

\section{Exercise testing}


maintain a cycling cadence of $>40 \mathrm{rpm}$. Concerning each of the three criteria for reaching the

114 maximal effort, 21 participants $(91.3 \%)$ had an increase in $\mathrm{VO}_{2}<150 \mathrm{~mL} \cdot \mathrm{min}^{-1}$ for $>1$ min

115 despite increased work rate, six (26.1\%) achieved a respiratory exchange ratio $>1.10$, and 11

$116(47.8 \%)$ reached $85 \%$ of the age-predicted maximal heart rate. The ventilatory threshold was

117 determined in all participants.

kinetics of $\dot{\mathrm{VO}}_{2}, \mathrm{CO}, \mathrm{AVO}_{2}$ diff, and minute ventilation $(\dot{\mathrm{V} E})$ were $0.99 \pm 0.01,0.96 \pm 0.02$,

$0.93 \pm 0.03$, and $0.98 \pm 0.01$, respectively. In addition, the mean $\pm \mathrm{SD}$ ratio of the time

constant of $\mathrm{CO}(\tau \mathrm{CO})$ to $\tau \dot{\mathrm{V}} \mathrm{O}_{2}$ was $1.19 \pm 0.56$. In 15 of 23 participants $(65.2 \%)$, the ratio of

$\tau \mathrm{CO}$ to $\tau \dot{\mathrm{V}} \mathrm{O}_{2}$ was $>1.00$.

Measurement values obtained during the symptom-limited graded and submaximal constant-load exercise tests are shown in Table 2. 
$38.6 \pm 10.1 \mathrm{~s}$, respectively.

The FSS score was not significantly correlated with $\dot{\mathrm{VO}}_{2}$ at peak exercise $(\mathrm{rho}=$ $-0.264, p=0.224 ;$ Fig. $2 a$ ), while a higher FSS score was significantly correlated with a longer $\tau \dot{\mathrm{V}} \mathrm{O}_{2}$ (rho $=0.530, \mathrm{p}=0.009$; Fig. 2b). Although the FSS score was not also associated with other cardiorespiratory variables at peak exercise and at the ventilatory threshold measured during the symptom-limited exercise test, a higher FSS score was significantly associated with a longer $\tau \mathrm{CO}(\mathrm{rho}=0.476, \mathrm{p}=0.022 ;$ Fig. 3$)$ during the submaximal constant-load exercise test. Changes in $\dot{\mathrm{V}}_{2}$ and $\mathrm{CO}$ at the onset of exercise in representative participants with different fatigue levels (low and high) are shown in Supplementary Figs. S1 and S2, respectively, online.

\section{Associations of participants' characteristics with the FSS score, $\tau \dot{\mathrm{V}} \mathrm{O}_{2}$, and $\tau \mathrm{CO}$} No demographic and clinical variables were significantly associated with the FSS score (Table 1) and $\tau \dot{\mathrm{V}} \mathrm{O}_{2}$ and $\tau \mathrm{CO}$ (Table 3). These results indicate that there were no potentially confounding variables in the associations of the FSS score with $\tau \dot{\mathrm{V}} \mathrm{O}_{2}$ and $\tau \mathrm{CO}$ measured during the exercise tests.

\section{Discussion}

The primary objective of this study was to examine whether more severe fatigue was 
associated with a longer $\tau \dot{\mathrm{V}} \mathrm{O}_{2}$ measured during a submaximal constant-load exercise test

rather than a lower $\dot{\mathrm{V}}_{2}$ at peak exercise obtained during a symptom-limited graded exercise test in inpatients at a subacute rehabilitation ward. The results of post-stroke fatigue assessment in this study are in line with those of previous studies [28,29]. This study demonstrated that a higher FSS score was associated with a longer $\tau \dot{\mathrm{V}} \mathrm{O}_{2}$ at the onset of exercise; however, fatigue was not associated with $\dot{\mathrm{V}}_{2}$ at peak exercise. In addition, our secondary objective was to identify the associations between post-stroke fatigue and cardiorespiratory variables potentially affecting $\dot{\mathrm{V}}_{2}$ during exercise, such as the OUES, CO, and $\mathrm{AVO}_{2}$ diff. As a result, a higher FSS score was associated with a longer $\tau \mathrm{CO}$ at the onset of exercise. As we observed no demographic and clinical variables significantly correlated with the FSS score, $\tau \dot{\mathrm{V}} \mathrm{O}_{2}$, and $\tau \mathrm{CO}$, this study indicated that the FSS score was associated with $\tau \dot{\mathrm{V}} \mathrm{O}_{2}$ and $\tau \mathrm{CO}$ independently of other participants' characteristics. Our findings suggest that the ability of the cardiorespiratory system to adapt to exercise is impaired in individuals with severe post-stroke fatigue. Individuals with post-stroke fatigue lack the energy necessary to perform activities, are more easily tired by activity, experience unpredictable and unexplainable feelings of fatigue, and have increased stress sensitivity and increased need for longer sleep durations, naps, or rest [30]. Thus, it is plausible that post-stroke fatigue might be associated with decreased cardiorespiratory fitness and reduced physical activity [12,31]. A systematic review 
reported that both post-stroke fatigue and reduced cardiorespiratory fitness were associated

fatigue that fatigue at the early stage after stroke may be attributed to biological factors [4],

all participants in the present study were recruited from a subacute rehabilitation ward.

However, our result regarding the association between the FSS score and $\dot{\mathrm{VO}}_{2}$ at peak

exercise is consistent with that in a previous study on chronic stroke [15]. To the best of our

knowledge, this is the first study to investigate the association between post-stroke fatigue

and $\tau \dot{\mathrm{V}} \mathrm{O}_{2}$ measured during the submaximal constant-load exercise test. We observed that the

that the fitting procedures were acceptable [33]. The mean value of $\tau \dot{\mathrm{V}} \mathrm{O}_{2}$ in this study was

had a longer $\tau \dot{\mathrm{V}} \mathrm{O}_{2}$ than age-, sex-, and activity-matched healthy adults [16]. In healthy adults, the acceleration of $\tau \dot{\mathrm{V}} \mathrm{O}_{2}$ has been reported to occur in the early period of endurance training, and $\dot{\mathrm{VO}}_{2}$ at peak exercise can subsequently increase [20,21]. Additionally, a previous study reported that $\tau \dot{\mathrm{V}} \mathrm{O}_{2}$ was shorter in the recreationally active group than in the inactive group; 
peak exercise in assessing cardiorespiratory functional changes, which may explain our

results that a higher FSS score was significantly correlated with a longer $\tau \dot{\mathrm{VO}}_{2}$ but not with

$\dot{\mathrm{VO}}_{2}$ at peak exercise.

192

We found that the mean ratio of $\tau \mathrm{CO}$ to $\tau \dot{\mathrm{V}} \mathrm{O}_{2}$ was $>1.00$, indicating that oxygen

delivery was not in excess of the metabolic demand during exercise onset and that $\dot{\mathrm{V}} \mathrm{O}_{2}$

kinetics at exercise onset were limited by a delayed increase in $\mathrm{CO}$ [34]. These findings may

explain the association between the FSS score and $\tau \mathrm{CO}$ observed in this study. Unfortunately,

because there have been no published studies that measured $\tau \mathrm{CO}$ in individuals with stroke, it

is unclear why participants with severe post-stroke fatigue showed a longer $\tau \mathrm{CO}$. However,

given that post-stroke fatigue is associated with low physical activity [32], an inactive

lifestyle may lead to a longer $\tau \mathrm{CO}$ in individuals with severe fatigue. The prompt increase in

$\mathrm{CO}$ at the onset of exercise is compatible with the notion of immediate vagal withdrawal.

after than before prolonged bed rest in healthy adults because of the decreased vagal activity

rest [38-41] may also negatively affect the increase in CO during exercise onset. In the future, 
individuals with fatigue at the early stage after stroke can contribute to the development of an appropriate rehabilitation program for these individuals.

$211 \tau \dot{\mathrm{V}} \mathrm{O}_{2}$ at the onset of exercise. Previous studies showed that aerobic exercise training was

212 effective in the improvement of $\tau \dot{\mathrm{V}} \mathrm{O}_{2}$ in older individuals $[22,42,43]$. In addition, a randomised controlled trial reported that the combination of cognitive-behavioural therapy

214 and graded activity training was more effective than cognitive-behavioural therapy alone in treating post-stroke fatigue [44]. Although post-stroke fatigue has a negative impact on the recovery of activities of daily living [5,6], a systematic review reported that aerobic exercise

217 can improve the functional ability in individuals with stroke [11]. Furthermore, at the

218 subacute phase of stroke recovery, several articles demonstrate the effectiveness of exercise

219 in improving health outcomes after stroke including cardiovascular, functional, and mobility

220 outcomes $[45,46]$. Therefore, because fatigue is associated with low levels of daily activities

221 after stroke [32], rehabilitative exercise programmes may be beneficial in individuals with

222 post-stroke fatigue. The use of exercise testing for the clinical assessment and exercise prescription is

224 limited in stroke rehabilitation settings [47,48], limiting the clinical applicability of our

225 findings. A lack of exercise equipment, time, space, and support staff have also been reported as a barrier to exercise testing [47]. In addition, the cardiac, cognitive, functional, and 
227 physical impairments in individuals with stroke may make it difficult to perform exercise testing safely [47]. More specific clinical guidelines for post-stroke exercise testing,

229 educational training associated with exercise testing, and greater collaboration between stroke

230 and cardiac rehabilitation teams can help implement exercise testing in stroke rehabilitation

231 settings [47,48].

This study had some limitations. First, the sample size was relatively small, although

it was determined based on a power analysis. The sum score of 36 or the average score of 4

234 was the cut-off value of the FSS score $[2,49]$. We could not examine whether the association

between the FSS score and cardiorespiratory variables during exercise could differ between

236 participants with and without post-stroke fatigue. In addition, although post-stroke fatigue has

237 been reported to be associated with clinical variables, such as depressive symptoms and

238 functional disability [1], these associations were not statistically significant. These results

239 may be attributed to a relatively small sample size. Furthermore, we could not perform the

240 subgroup analyses according to sex, the phase of stroke recovery, and the type of stroke of the

241 participants. Being female has been reported to be associated with the presence of post-stroke

242 fatigue and a longer $\tau \dot{\mathrm{V}} \mathrm{O}_{2}[1,50]$. Changes in the brain after stroke may also affect post-stroke

243 fatigue and cardiorespiratory control during exercise [1,51]. Even though our participants

244 were recruited from a subacute rehabilitation ward, five participants were in the chronic

245 phase of stroke recovery [27]. In addition, 12 participants with ischaemic stroke and 11 with 
246 haemorrhagic stroke were included in this study. Functional recovery differs between

247 individuals with ischaemic and haemorrhagic stroke. Stroke severity is higher in

248 haemorrhagic stroke than in ischaemic stroke, while individuals with haemorrhagic stroke

249 have been shown to have a higher therapeutic response to rehabilitation than those with

250 ischaemic stroke [52,53]. This study demonstrated that sex, the time since stroke, and the

251 type of stroke were not confounding variables in the associations of the FSS score with $\tau \dot{\mathrm{V}} \mathrm{O}_{2}$

252 and $\tau$ CO. Nevertheless, further studies employing a stratified sampling method would

253 increase the generalisability of our results.

255 fatigue present over 1 year after stroke may be more associated with psychological and

256 behavioural factors than biological factors [4], the generalisation of our findings to

257 individuals who are over 1 year after stroke should be made with caution. Third, many

258 individuals with stroke $(\mathrm{n}=424)$ were excluded from the study. Many of them were excluded

259 due to being $>80$ years of age, a Mini-Mental State Examination score $\leq 24$ points, and/or

260 unstable medical conditions, as shown in Fig.1. This may limit the generalisability of our

261 findings to individuals with these conditions. Finally, because this study used a cross-

262 sectional observational design, the cardiorespiratory variables associated with temporal

263 changes in post-stroke fatigue could not be examined. Thus, further longitudinal studies are

264 needed to investigate the temporal association between post-stroke fatigue and 
cardiorespiratory fitness variables.

$267 \tau \dot{\mathrm{V}} \mathrm{O}_{2}$ at the onset of exercise measured during a submaximal constant-load exercise test, but

268 not with $\dot{\mathrm{V}} \mathrm{O}_{2}$ at peak exercise obtained during a symptom-limited graded exercise test. In

269 addition, a higher FSS score was associated with a longer $\tau \mathrm{CO}$ at the onset of exercise. These

270 results suggest that the ability of the cardiorespiratory system to adapt to exercise is impaired

271 in individuals with severe post-stroke fatigue. Collectively, our findings can contribute to the

272 development of an appropriate rehabilitation program for individuals with post-stroke fatigue.

\section{Study design}

This study used a cross-sectional design. The study protocol was approved by the

277 appropriate ethics committees of Tokyo Bay Rehabilitation Hospital (approval number, 172-

278 2) and Shinshu University (approval number: 3813). All participants provided written

279 informed consent before their enrolment in the study. The study was conducted according to 280 the Declaration of Helsinki of 1964, as revised in 2013.

\section{Participants}



as age and the type of stroke, were obtained from the patients' medical record.

\section{Procedure} the second day, participants performed a symptom-limited graded exercise test to determine 
measure of post-stroke fatigue [2]. Each item was rated on a 7-point Likert scale that ranged

304 from 1 to 7 (1, strongly disagree; 7, strongly agree). The FSS score was calculated as the sum of 9-item scores. A higher score indicated a large impact of fatigue on daily activities. The 15-item Geriatric Depression Scale (GDS) [56] was used to assess depressive symptoms. The presence of depressive symptoms was denoted by a GDS score $\geq 5$ points.

The motor function and independency in performing daily activities were assessed as

functional outcomes. The total Stroke Impairment Assessment Set motor function score was measured to assess motor impairments in the paretic upper and lower extremities [57]. The Functional Independence Measure score was used to evaluate the degree of independence in activities of daily living [58].

\section{Exercise testing}

316 for at least $6 \mathrm{~h}$ and vigorous physical activity for $24 \mathrm{~h}$ before the symptom-limited graded and

317 submaximal constant-load exercise tests [59]. These tests were performed on a recumbent

318 cycle ergometer (Strength Ergo 240; Mitsubishi Electric Engineering Co., Ltd., Tokyo, Japan)

319 that could be precisely load-controlled (coefficient of variation, 5\%) over a wide range of

320 pedalling resistance $(0-400 \mathrm{~W})$. Participants were instructed to maintain a target cadence of

$32150 \mathrm{rpm}$ in all exercise phases [59]. Expired gas was measured on a breath-by-breath basis 
322 during the exercise test using an expired gas analyser (Aerosonic AT-1100; ANIMA Corp.,

323 Tokyo, Japan). Before expired gas data collection, the analyser was calibrated using gas

324 mixtures with accurately known concentrations of oxygen and carbon dioxide. CO was

325 measured on a beat-by-beat basis using a noninvasive impedance cardiography device (Task

326 Force Monitor model 3040i; CN Systems Medizintechnik GmbH., Graz, Austria), as

327 previously described [60]. Three short band electrodes, one on the neck and two others below

328 the thorax, were placed on participants. Stroke volume was calculated with the following

329 equation:

330

Stroke volume $=\mathrm{V}_{\text {th }} \times$ LVET $\times(\mathrm{dZ} / \mathrm{dt})_{\max } / \mathrm{Z}_{0}$

331

where $\mathrm{V}_{\text {th }}$ is the electrical participating thoracic volume, LVET is the left ventricular ejection

time, $(\mathrm{dZ} / \mathrm{dt})_{\max }$ is the maximal rate of decrease in impedance for a given heartbeat, and $\mathrm{Z}_{0}$ is

the base impedance. $\mathrm{CO}$ was calculated as the product of stroke volume and heart rate. The

impedance cardiography method is a valid and reliable method for measuring cardiac

haemodynamics at rest and during exercise [60]. The measurement values of

$5 \mathrm{~s}$ bins to derive the $\mathrm{AVO}_{2}$ diff on a second-by-second basis [16,24], which was calculated as

$$
\dot{\mathrm{V}} \mathrm{O}_{2}=\mathrm{CO} \times \mathrm{AVO}_{2} \text { diff. }
$$


341 limited graded exercise test started with a warm-up at $0 \mathrm{~W}$ for $3 \mathrm{~min}$ followed by a $10-\mathrm{W}$

342 increment every minute [59]. The test was terminated if the participant showed signs of

343 angina, dyspnoea, inability to maintain a cycling cadence of more than $40 \mathrm{rpm}$, hypertension

344 ( $>250 \mathrm{mmHg}$ systolic or $>115 \mathrm{mmHg}$ diastolic), or a drop in systolic blood pressure by $>10$

$345 \mathrm{mmHg}$, despite the increase in workload [24]. To identify whether the maximal effort was

346 reached during the exercise test, at least one of the following criteria had to be met: $\dot{\mathrm{VO}}_{2}$

347 increased $<150 \mathrm{~mL} \cdot \mathrm{min}^{-1}$ for $>1$ min despite increased work rate, respiratory exchange ratio

$348>1.10$, or heart rate achieved $85 \%$ of the age-predicted maximal heart rate calculated as 220

349 minus age [14]. $\dot{\mathrm{V}} \mathrm{O}_{2}, \mathrm{CO}, \mathrm{AVO}_{2}$ diff, $\dot{\mathrm{V}}$, and respiratory exchange ratio at peak exercise

350 were defined as the average value obtained during the last $30 \mathrm{~s}$ of the exercise test [59]. In

351 addition, the OUES was determined by calculating the slope of the regression line between

$352 \dot{\mathrm{V}} \mathrm{O}_{2}$ and the log transformation of $\dot{\mathrm{V}} \mathrm{E}$ during the whole exercise period using the following

353 equation:

354

$$
\dot{\mathrm{V}} \mathrm{O}_{2}=a \log \dot{\mathrm{V}} \mathrm{E}+b
$$

355

where the constant $a$ is the OUES [62]. A low OUES represents a higher amount of

ventilation required in response to a given oxygen uptake, which indicates ventilatory

inefficiency during exercise.

The ventilatory threshold was determined using a combination of the following

criteria: the point where the ventilatory equivalent of oxygen reaches its minimum or starts to 
360 increase, without an increase in the ventilatory equivalent of carbon dioxide; the point at

361 which the end-tidal oxygen fraction reaches a minimum or starts to increase, without a

362 decline in the end-tidal carbon dioxide fraction; and the point of deflection of carbon dioxide

363 output versus $\dot{\mathrm{V}} \mathrm{O}_{2}$ (the $\mathrm{V}$-slope method), as previously described [24,63]. $\dot{\mathrm{V}} \mathrm{O}_{2}, \mathrm{CO}$,

$364 \mathrm{AVO}_{2}$ diff, $\dot{\mathrm{V} E}$, and respiratory exchange ratio at the ventilatory threshold were obtained.

The submaximal constant-load exercise test started with resting on the cycle

ergometer for $3 \mathrm{~min}$, followed by performing exercise at $80 \%$ of the workload corresponding

to the ventilatory threshold for 6 min $[19,34]$. The protocol was repeated three times, with a

rest between each repetition. The kinetics of the $\dot{\mathrm{V}} \mathrm{O}_{2}, \mathrm{CO}, \mathrm{AVO}_{2}$ diff, and $\dot{\mathrm{V}} \mathrm{E}$ data at the

onset of exercise were obtained by averaging the three repeats. Additionally, before

modelling, the first $20 \mathrm{~s}$ of data after the onset of the exercise was eliminated, as during this

period, the increase in $\dot{\mathrm{V}}_{2}$ reflects merely an increase in the pulmonary blood flow rather

than changes in tissue gas exchange [18]. To calculate the time constants of $\dot{\mathrm{V}} \mathrm{O}_{2}, \mathrm{CO}$,

$373 \mathrm{AVO}_{2} \mathrm{diff}$, and $\dot{\mathrm{V} E}$ at the onset of exercise, a non-linear least squares regression procedure

374 (GraphPad Prism version 7.00 for Windows; GraphPad Software, CA, USA) was applied to

375 the onset phase, using the following formula:

$$
\mathrm{Y}(\mathrm{t})=\mathrm{Y}_{\text {baseline }}+\left(\mathrm{Y}_{\text {steady-state }}-\mathrm{Y}_{\text {baseline }}\right) *\left(1-\exp ^{-(\mathrm{t}-\mathrm{TD}) / \tau}\right)
$$

377 where $\mathrm{Y}(\mathrm{t})$ represents $\dot{\mathrm{V}} \mathrm{O}_{2}, \mathrm{CO}, \mathrm{AVO}_{2}$ diff, or $\dot{\mathrm{VE}}$ at a given time (t); $\mathrm{TD}$ is the time delay; 
379 during the last minute of the resting period and exercise, respectively. Fit quality was

380 determined by the coefficient of determination. The fitting procedure was considered

381 acceptable if the coefficient of determination was $>0.85$ [33]. In addition, the ratio of $\tau \mathrm{CO}$ to

$382 \tau \dot{\mathrm{V}} \mathrm{O}_{2}>1.00$ indicated a slow increase in $\mathrm{CO}$ relative to $\dot{\mathrm{V}} \mathrm{O}_{2}$ at the onset of exercise.

\section{Statistical analyses}

The sample size for correlational analysis was computed for 0.05 alpha, 0.80 power,

and 0.50 estimated effect size (large) [15] with G Power software version 3.1.9.2 (Heinrich

Heine University, Dusseldorf, Germany). Consequently, a minimum sample size of 26

participants was required. Assuming $10 \%$ of the participants could be excluded, we aimed to

recruit 30 participants.

The results are shown as medians (interquartile ranges) or means \pm SDs. We

examined the associations between the FSS score and cardiorespiratory variables measured

during the exercise tests using the Spearman's rank correlation coefficient. To identify the

potentially confounding variables, we also determined the associations of participants'

characteristics with the FSS score and cardiorespiratory variables that significantly correlated

with the FSS score using the Pearson's product-moment correlation coefficient, the

Spearman's rank correlation coefficient, and the unpaired t-test based on variable types. 
398 Computing, Vienna, Austria). Poisson (p) values $<0.05$ were considered statistically

399 significant. 


\section{Data availability}

401 The datasets generated during and/or analysed during the current study are available

402 from the corresponding author on reasonable request.

403

\section{References}

405

1. Hinkle, J. L. et al. Poststroke fatigue: emerging evidence and approaches to management: a

406

407

408

409

410

2. Cumming, T. B., Packer, M., Kramer, S. F. \& English, C. The prevalence of fatigue after scientific statement for healthcare professionals from the American Heart Association.

Stroke 48, e159-e170, https://doi.org/10.1161/STR.0000000000000132 (2017).

stroke: A systematic review and meta-analysis. Int J Stroke 11, 968-977, https://doi.org/10.1177/1747493016669861 (2016).

3. Lynch, J. et al. Fatigue after stroke: the development and evaluation of a case definition. $J$

4. Wu, S., Mead, G., Macleod, M. \& Chalder, T. Model of understanding fatigue after stroke.

5. Lerdal, A. \& Gay, C. L. Acute-phase fatigue predicts limitations with activities of daily

416 living 18 months after first-ever stroke. J Stroke Cerebrovasc Dis 26, 523-531,

417 https://doi.org/10.1016/j.jstrokecerebrovasdis.2016.11.130 (2017). 

stroke patients in Sweden. Stroke 33, 1327-1333 (2002).

420

421

422

423

424

425

426

427

428

429

430

431

432

433

434

435

436

437

7. Pihlaja, R., Uimonen, J., Mustanoja, S., Tatlisumak, T. \& Poutiainen, E. Post-stroke fatigue is associated with impaired processing speed and memory functions in first-ever stroke patients. J Psychosom Res 77, 380-384, https://doi.org/10.1016/j.jpsychores.2014.08.011 (2014).

8. Lerdal, A. \& Gay, C. L. Fatigue in the acute phase after first stroke predicts poorer physical health 18 months later. Neurology 81, 1581-1587, https://doi.org/10.1212/WNL.0b013e3182a9f471 (2013).

9. Naess, H. \& Nyland, H. Poststroke fatigue and depression are related to mortality in young adults: a cohort study. BMJ Open 3, https://doi.org/10.1136/bmjopen-2012-002404 (2013).

10. McGeough, E. et al. Interventions for post-stroke fatigue. Cochrane Database Syst Rev, CD007030, https://doi.org/10.1002/14651858.CD007030.pub2 (2009).

11. Saunders, D. H. et al. Physical fitness training for stroke patients. Cochrane Database Syst Rev 3, CD003316, https://doi.org/10.1002/14651858.CD003316.pub7 (2020).

12. Duncan, F., Kutlubaev, M. A., Dennis, M. S., Greig, C. \& Mead, G. E. Fatigue after stroke: a systematic review of associations with impaired physical fitness. Int J Stroke 7, 157-162, https://doi.org/10.1111/j.1747-4949.2011.00741.x (2012).

13. Smith, A. C., Saunders, D. H. \& Mead, G. Cardiorespiratory fitness after stroke: a systematic review. Int J Stroke 7, 499-510, https://doi.org/10.1111/j.1747- 

4949.2012.00791.x (2012).

439

440

441

442

443

444

445

446

447

448

449

450

451

452

453

454

455

456

14. Wittink, H. et al. Measurement properties of maximal cardiopulmonary exercise tests protocols in persons after stroke: a systematic review. J Rehabil Med 49, 689-699, https://doi.org/10.2340/16501977-2260 (2017).

15. Tseng, B. Y., Billinger, S. A., Gajewski, B. J. \& Kluding, P. M. Exertion fatigue and chronic fatigue are two distinct constructs in people post-stroke. Stroke 41, 2908-2912, https://doi.org/10.1161/STROKEAHA.110.596064 (2010).

16. Tomczak, C. R. et al. Cardiac reserve and pulmonary gas exchange kinetics in patients with stroke. Stroke 39, 3102-3106, https://doi.org/10.1161/STROKEAHA.108.515346 (2008).

17. Manns, P. J., Tomczak, C. R., Jelani, A. \& Haennel, R. G. Oxygen uptake kinetics: associations with ambulatory activity and physical functional performance in stroke survivors. J Rehabil Med 42, 259-264, https://doi.org/10.2340/16501977-0498 (2010).

18. Poole, D. C. \& Jones, A. M. Oxygen uptake kinetics. Compr Physiol 2, 933-996, https://doi.org/10.1002/cphy.c100072 (2012).

19. George, M. A., McLay, K. M., Doyle-Baker, P. K., Reimer, R. A. \& Murias, J. M. Fitness level and not aging per se, determines the oxygen uptake kinetics response. Front Physiol 9, 277, https://doi.org/10.3389/fphys.2018.00277 (2018).

20. Phillips, S. M., Green, H. J., MacDonald, M. J. \& Hughson, R. L. Progressive effect of 
endurance training on VO2 kinetics at the onset of submaximal exercise. J Appl Physiol (1985) 79, 1914-1920, https://doi.org/10.1152/jappl.1995.79.6.1914 (1995).

21. Fukuoka, Y. et al. Early effects of exercise training on on- and off-kinetics in 50-year-old subjects. Pflugers Arch 443, 690-697, https://doi.org/10.1007/s00424-001-0748-y (2002).

22. Hamasaki, A., Arima, S. \& Hirakoba, K. Changes in pulmonary oxygen uptake and muscle deoxygenation kinetics during cycling exercise in older women performing walking training for 12 weeks. Eur J Appl Physiol 118, 2179-2188, https://doi.org/10.1007/s00421-018-3946-4 (2018).

23. Sisante, J. F., Mattlage, A. E., Arena, R., Rippee, M. A. \& Billinger, S. A. Decreased tidal volume may limit cardiopulmonary performance during exercise in subacute stroke. $J$ Cardiopulm Rehabil Prev 35, 334-341, https://doi.org/10.1097/HCR.0000000000000119 (2015).

24. Oyake, K. et al. Cardiorespiratory factors related to the increase in oxygen consumption during exercise in individuals with stroke. PLoS One 14, e0217453, https://doi.org/10.1371/journal.pone.0217453 (2019).

25. Baert, I. et al. Evolution of cardiorespiratory fitness after stroke: a 1-year follow-up study. Influence of prestroke patients' characteristics and stroke-related factors. Arch Phys Med Rehabil 93, 669-676, https://doi.org/10.1016/j.apmr.2011.09.022 (2012).

26. Jakovljevic, D. G. et al. Discrepancy between cardiac and physical functional reserves in 
27. Sullivan, K. J. On "Modified constraint-induced therapy...” Page and Levine. Phys Ther. 2007;87:872-878. Phys Ther 87, 1560-1560, https://doi.org/10.2522/ptj.2007.87.11.1560 (2007).

28. van de Port, I. G., Wevers, L. E., Lindeman, E. \& Kwakkel, G. Effects of circuit training e2672, https://doi.org/10.1136/bmj.e2672 (2012).

29. Harbison, J. A., Walsh, S. \& Kenny, R. A. Hypertension and daytime hypotension found 102, 109-115, https://doi.org/10.1093/qjmed/hen146 (2009).

30. Eilertsen, G., Ormstad, H. \& Kirkevold, M. Experiences of poststroke fatigue: qualitative

32. Thilarajah, S. et al. Factors associated with post-stroke physical activity: a systematic review and meta-analysis. Arch Phys Med Rehabil 99, 1876-1889, https://doi.org/10.1016/j.apmr.2017.09.117 (2018). 
33. Kemps, H. M. et al. Reproducibility of onset and recovery oxygen uptake kinetics in moderately impaired patients with chronic heart failure. Eur J Appl Physiol 100, 45-52, https://doi.org/10.1007/s00421-007-0398-7 (2007).

34. Kemps, H. M. et al. Are oxygen uptake kinetics in chronic heart failure limited by oxygen delivery or oxygen utilization? Int J Cardiol 142, 138-144, https://doi.org/10.1016/j.ijcard.2008.12.088 (2010).

35. Capelli, C., Adami, A., Antonutto, G., Cautero, M. \& Tam, E. Oxygen deficits and oxygen delivery kinetics during submaximal intensity exercise in humans after 14 days of headdown tilt-bed rest. Eur J Appl Physiol 107, 51-59, https://doi.org/10.1007/s00421-0091098-2 (2009).

36. Al-Qudah, Z. A., Yacoub, H. A. \& Souayah, N. Disorders of the autonomic nervous system after hemispheric cerebrovascular disorders: an update. $J$ Vasc Interv Neurol $\mathbf{8}$, 43-52 (2015).

37. Lees, T. et al. Heart rate variability as a biomarker for predicting stroke, post-stroke complications and functionality. Biomark Insights 13, 1177271918786931, https://doi.org/10.1177/1177271918786931 (2018).

38. Perhonen, M. A. et al. Cardiac atrophy after bed rest and spaceflight. J Appl Physiol (1985) 91, 645-653, https://doi.org/10.1152/jappl.2001.91.2.645 (2001). 
remodeling during and after 60 days of sedentary head-down bed rest. J Appl Physiol (1985) 120, 956-964, https://doi.org/10.1152/japplphysiol.00676.2015 (2016).

40. Chobanian, A. V., Lille, R. D., Tercyak, A. \& Blevins, P. The metabolic and hemodynamic https://doi.org/10.1161/01.cir.49.3.551 (1974).

519

520

521

522

523

524

525

526

527

528

529

530

531

532

effects of prolonged bed rest in normal subjects. Circulation 49, 551-559,

41. Convertino, V. A. Cardiovascular consequences of bed rest: effect on maximal oxygen uptake. Med Sci Sports Exerc 29, 191-196, https://doi.org/10.1097/00005768199702000-00005 (1997).

42. Murias, J. M., Kowalchuk, J. M. \& Paterson, D. H. Speeding of VO2 kinetics in response to endurance-training in older and young women. Eur J Appl Physiol 111, 235-243, https://doi.org/10.1007/s00421-010-1649-6 (2011).

43. Murias, J. M., Kowalchuk, J. M. \& Paterson, D. H. Speeding of VO2 kinetics with endurance training in old and young men is associated with improved matching of local O2 delivery to muscle O2 utilization. J Appl Physiol (1985) 108, 913-922, https://doi.org/10.1152/japplphysiol.01355.2009 (2010).

44. Zedlitz, A. M., Rietveld, T. C., Geurts, A. C. \& Fasotti, L. Cognitive and graded activity training can alleviate persistent fatigue after stroke: a randomized, controlled trial. Stroke 43, 1046-1051, https://doi.org/10.1161/strokeaha.111.632117 (2012).

45. Stoller, O., de Bruin, E. D., Knols, R. H. \& Hunt, K. J. Effects of cardiovascular exercise 
early after stroke: systematic review and meta-analysis. BMC Neurol 12, 45, https://doi.org/10.1186/1471-2377-12-45 (2012).

535

46. Billinger, S. A. et al. Aerobic exercise in subacute stroke improves cardiovascular health and physical performance. J Neurol Phys Ther 36, 159-165, https://doi.org/10.1097/NPT.0b013e318274d082 (2012).

47. Moncion, K. et al. Barriers and facilitators to aerobic exercise implementation in stroke rehabilitation: a scoping review. J Neurol Phys Ther 44, 179-187, https://doi.org/10.1097/npt.0000000000000318 (2020).

48. Boyne, P. et al. Aerobic exercise prescription in stroke rehabilitation: a web-based survey of US physical therapists. J Neurol Phys Ther 41, 119-128, https://doi.org/10.1097/npt.0000000000000177 (2017).

49. Krupp, L. B., LaRocca, N. G., Muir-Nash, J. \& Steinberg, A. D. The fatigue severity scale. Application to patients with multiple sclerosis and systemic lupus erythematosus. Arch Neurol 46, 1121-1123 (1989).

50. Franco, R. L. et al. Sex differences in pulmonary oxygen uptake kinetics in obese adolescents. J Pediatr 165, 1161-1165, https://doi.org/10.1016/j.jpeds.2014.08.005 (2014).

51. Billinger, S. A., Coughenour, E., Mackay-Lyons, M. J. \& Ivey, F. M. Reduced cardiorespiratory fitness after stroke: biological consequences and exercise-induced 

adaptations. Stroke Res Treat 2012, 959120, https://doi.org/10.1155/2012/959120 (2012).

553

554

555

556

557

558

559

560

561

562

563

564

565

566

567

568

569

570

52. Andersen, K. K., Olsen, T. S., Dehlendorff, C. \& Kammersgaard, L. P. Hemorrhagic and ischemic strokes compared: stroke severity, mortality, and risk factors. Stroke 40, 20682072, https://doi.org/10.1161/strokeaha.108.540112 (2009).

53. Paolucci, S. et al. Functional outcome of ischemic and hemorrhagic stroke patients after inpatient rehabilitation: a matched comparison. Stroke 34, 2861-2865, https://doi.org/10.1161/01.str.0000102902.39759.d3 (2003).

54. Folstein, M. F., Folstein, S. E. \& McHugh, P. R. "Mini-mental state". A practical method for grading the cognitive state of patients for the clinician. J Psychiatr Res 12, 189-198 (1975).

55. Spencer, M. D., Murias, J. M., Lamb, H. P., Kowalchuk, J. M. \& Paterson, D. H. Are the parameters of $\mathrm{VO} 2$, heart rate and muscle deoxygenation kinetics affected by serial moderate-intensity exercise transitions in a single day? Eur J Appl Physiol 111, 591-600, https://doi.org/10.1007/s00421-010-1653-x (2011).

56. Sheikh, J. I. Geriatric Depression Scale (GDS). Recent evidence and development of a shorter version. Clin Gerontol 5, 165-173, https://doi.org/10.1300/J018v05n01_09 (1986).

57. Chino, N., Sonoda, S., Domen, K., Saitoh, E. \& Kimura, A. Stroke Impairment Assessment Set (SIAS) A new evaluation instrument for stroke patients. Jpn J Rehabil 
Med 31, 119-125, https://doi.org/10.2490/jjrm1963.31.119 (1994).

572

58. Granger, C. V., Hamilton, B. B., Linacre, J. M., Heinemann, A. W. \& Wright, B. D. Performance profiles of the functional independence measure. Am J Phys Med Rehabil 72, 84-89, doi:10.1097/00002060-199304000-00005 (1993).

59. van de Port, I. G., Kwakkel, G. \& Wittink, H. Systematic review of cardiopulmonary exercise testing post stroke: Are we adhering to practice recommendations? J Rehabil Med 47, 881-900, doi:10.2340/16501977-2031 (2015).

60. Fortin, J. et al. Non-invasive beat-to-beat cardiac output monitoring by an improved method of transthoracic bioimpedance measurement. Comput Biol Med 36, 1185-1203, https://doi.org/10.1016/j.compbiomed.2005.06.001 (2006).

61. Balady, G. J. et al. Clinician's guide to cardiopulmonary exercise testing in adults: a scientific statement from the American Heart Association. Circulation 122, 191-225, https://doi.org/10.1161/CIR.0b013e3181e52e69 (2010).

62. Baba, R. et al. Oxygen uptake efficiency slope: a new index of cardiorespiratory functional reserve derived from the relation between oxygen uptake and minute ventilation during incremental exercise. J Am Coll Cardiol 28, 1567-1572, https://doi.org/10.1016/s0735-1097(96)00412-3 (1996).

63. Binder, R. K. et al. Methodological approach to the first and second lactate threshold in incremental cardiopulmonary exercise testing. Eur J Cardiovasc Prev Rehabil 15, 726- 


\section{Acknowledgments}

593

The authors certify that no other persons have made substantial contributions to this

594

manuscript. This work was supported by a grant from the Funds for a Grant-in-Aid for Young

595 Scientists to Kazuaki Oyake (18K17730). The funding source had no involvement with the

596 study design; collection, analysis, and interpretation of data; writing of the report; and the

597 decision to submit the article for publication.

598

599

Author contributions

600

KO conceptualised and designed the study, carried out the data analysis and

601

interpretation, and drafted the submitted article. YB, YS, JM, AM, YI, and HA designed the

602

study, coordinated subject recruitment, and performed data collection. YO and KK designed

603

the study, carried out the data analysis and interpretation, and revised the final version. KM

604

conceptualised and designed the study, carried out the data analysis and interpretation, and

605

revised the final version. All authors approved the final version.

606

607

\section{Competing interests}

608

The authors declare no competing interests.

609 


\section{Figure legends}

611

612 Figure 1. Flow diagram of study participants. MMSE, Mini-Mental State Examination.

613

614 Figure 2. Correlations of the Fatigue Severity Scale score with (a) oxygen uptake at peak

615 exercise and (b) the time constant of oxygen uptake kinetics.

616

617 Figure 3. Correlations of the Fatigue Severity Scale score and the time constant of cardiac

618 output kinetics. 


\section{Figures}

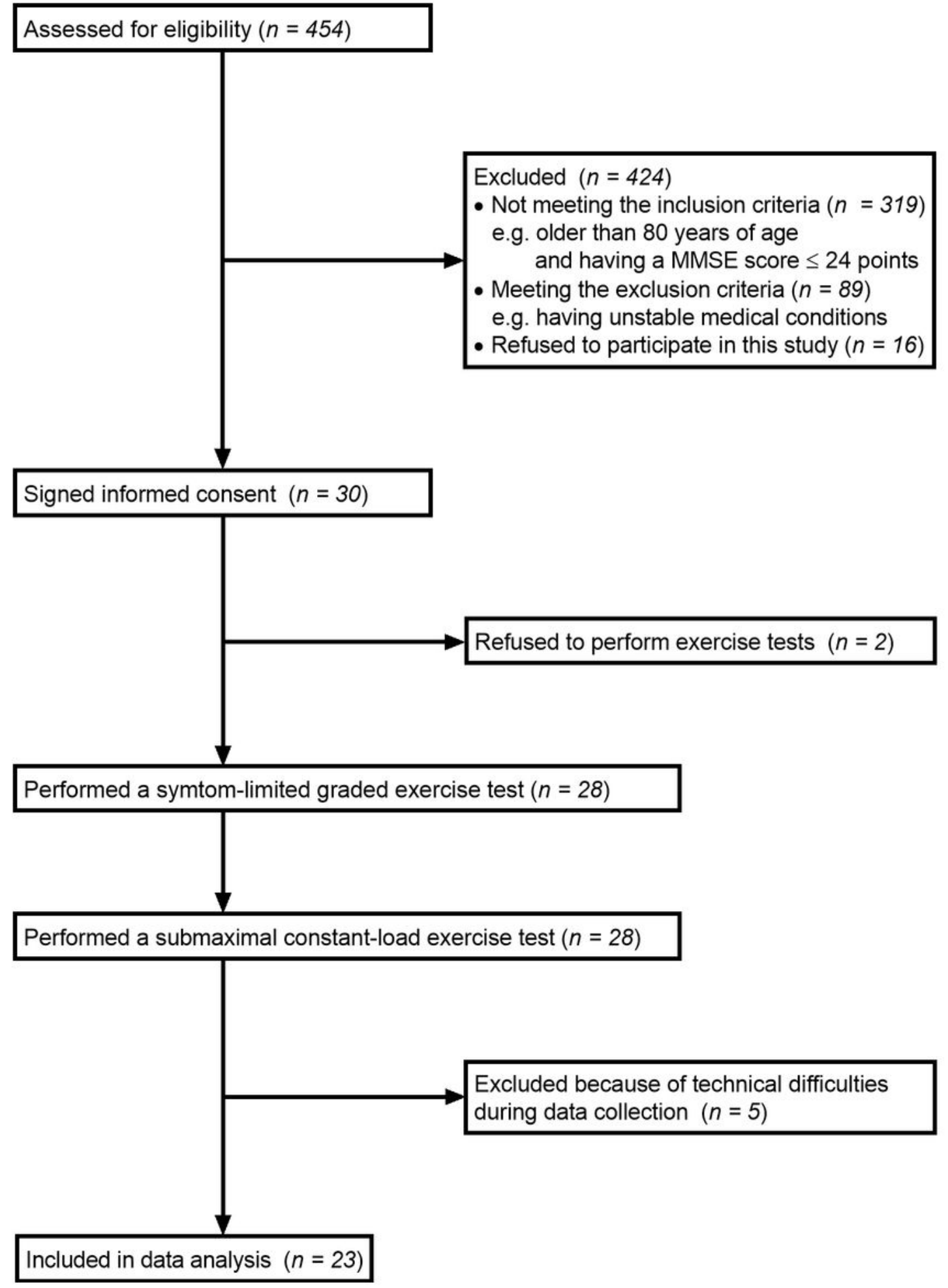

\section{Figure 1}

Flow diagram of study participants. MMSE, Mini-Mental State Examination. 

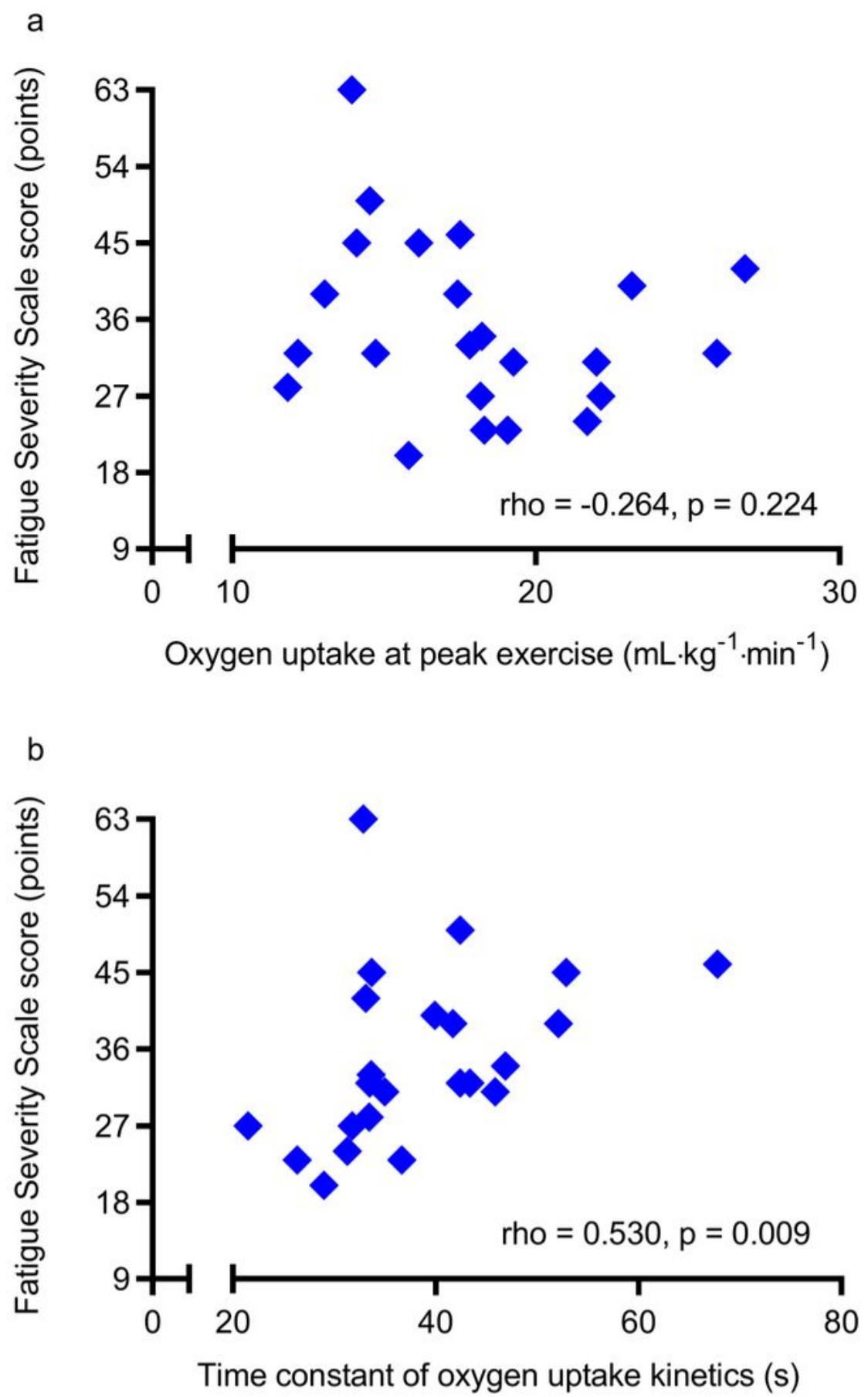

Figure 2

Correlations of the Fatigue Severity Scale score with (a) oxygen uptake at peak exercise and (b) the time constant of oxygen uptake kinetics. 


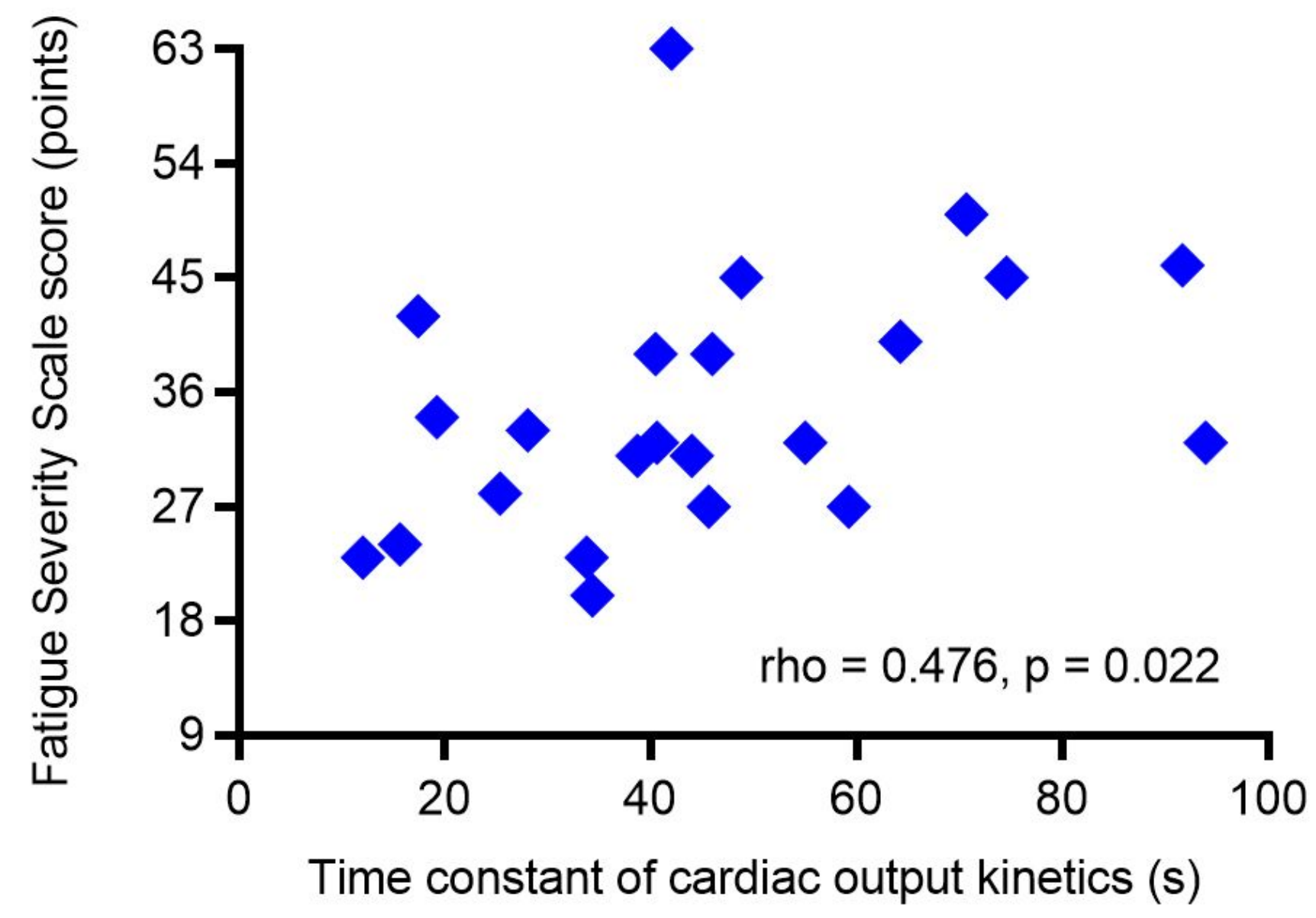

Figure 3

Correlations of the Fatigue Severity Scale score and the time constant of cardiac output kinetics.

\section{Supplementary Files}

This is a list of supplementary files associated with this preprint. Click to download.

- SupplementaryInformationpdfrevision.pdf 